J. theor. Biol. (2002) 218, 187-194

doi:10.1006/yjtbi.3067, available online at http://www.idealibrary.com on IDE $\mathbf{A}^{\circledR}$

\title{
Replicator Dynamics for Optional Public Good Games
}

\author{
Christoph Hauert $\dagger \dagger$, Silvia De Monte $\nmid \S$, Josef Hofbauer $\dagger$ and \\ KARL SIGMUND*†\|

\begin{abstract}
$\dagger$ Institute for Mathematics, University of Vienna, Strudlhofgasse 4, A-1090 Vienna, Austria $\$$ Department of Zoology, University of British Columbia, 6270 University Boulevard, Vancouver, BC, Canada V6T $1 Z 4$ § Department of Physics, Danish Technical University, DK-2800 Kgs. Lyngby, Denmark and \|11ASA, A-2361 Laxenburg, Austria
\end{abstract}

(Received on 8 October 2001, Accepted in revised form on 12 April 2002)

\begin{abstract}
The public goods game represents a straightforward generalization of the prisoner's dilemma to an arbitrary number of players. Since the dominant strategy is to defect, both classical and evolutionary game theory predict the asocial outcome that no player contributes to the public goods. In contrast to the compulsory public goods game, optional participation provides a natural way to avoid deadlocks in the state of mutual defection. The three resulting strategies - collaboration or defection in the public goods game, as well as not joining at all - are studied by means of a replicator dynamics, which can be completely analysed in spite of the fact that the payoff terms are nonlinear. If cooperation is valuable enough, the dynamics exhibits a rock-scissors-paper type of cycling between the three strategies, leading to sizeable average levels of cooperation in the population. Thus, voluntary participation makes cooperation feasible. But for each strategy, the average payoff value remains equal to the earnings of those not participating in the public goods game.
\end{abstract}

(C) 2002 Elsevier Science Ltd. All rights reserved.

\section{Introduction}

Most theories on the emergence of cooperation among selfish individuals are based on kin selection (Hamilton, 1963), group selection (Wilson \& Sober, 1994) and reciprocal altruism (Trivers, 1971). In all three models, cooperative behavior is attained through basic mechanisms of discrimination enabling individuals to target their altruistic acts towards certain partners only.

\footnotetext{
*Corresponding author. Institute for Mathematics, University of Vienna, Strudlhofgasse 4, A-1090 Vienna, Austria. Tel.: +43-3-4277-50612; fax: +43-1-4277-9506.

E-mail address: karl.sigmund@univie.ac.at (K. Sigmund).
}

In this article, we present another mechanism to achieve sizeable levels of cooperation in a population. The following investigation is based on the public goods game (see Fehr \& Gächter, 2002; Kagel \& Roth, 1995) which represents a natural extension of the prisoner's dilemma to an arbitrary number of players (see Boyd \& Richerson, 1988; Dawes, 1980; Hauert \& Schuster, 1997).

In a typical public goods game, an experimenter gives 20 dollars to each of eight players. The players may contribute part or all of their money to some common pool. The experimenter then triples this amount and divides it equally among the eight players, irrespective of the amount of their individual contribution. If all players contribute maximally, they will end up 
with 60 dollars each. But each individual is faced with the temptation to exploit, as a free rider, the contributions of the co-players. Hence, the dominating strategy is to invest nothing at all. If all players do this, they will not increase their initial capital. In this sense, the "rational" equilibrium solution prescribed to "homo oeconomicus" leads to economic stalemate. In actual experiments, players tend to invest a lot, however: typically, in the first round, they invest 10 dollars or more (Fehr \& Gächter, 2002).

Public goods games are abundant in human and animal societies, and can be seen as basic examples of economic interactions (see e.g. Binmore, 1994; Dugatkin, 1997).

\section{The Model}

We consider a large population of players. From time to time, $N$ such players are chosen randomly. Within such a group, players can either contribute some fixed amount $c$ or nothing at all. The return of the public good, i.e. the payoff to the players in the group, depends on the abundance of cooperators. If $n_{c}$ denotes their number among the public goods players, the net payoff for cooperators $P_{c}$ and defectors $P_{d}$ is given by

$$
\begin{gathered}
P_{c}=-c+r c \frac{n_{c}}{N}, \\
P_{d}=r c \frac{n_{c}}{N},
\end{gathered}
$$

where $r$ denotes the interest rate on the common pool. For a public goods game deserving its name, we must have

$$
1<r<N
$$

The first inequality states that if all do the same, they are better off cooperating than defecting; the second inequality states that each individual is better off defecting than cooperating. Selfish players will therefore always avoid the cost of cooperation $c$, i.e. a collective of selfish players will never cooperate. Defection is the dominating strategy. Hence both classical and evolutionary game theory predict that all players will defect, and obtain zero payoff.

We now extend the public goods game. In this optional public goods game, players can decide whether to participate in the public goods game or not. [For a similar approach in the prisoner's dilemma see Batali \& Kitcher (1995); Orbell \& Dawes (1993)]. Individuals unwilling to join the public goods game are termed loners. These players prefer to rely on a small but fixed payoff $P_{l}=\sigma c$ with

$$
0<\sigma<r-1
$$

such that the members in a group where all cooperate are better off than loners, but loners are better off than members in a group of defectors.

For the optional public goods game, there are thus three behavioral types in the population: (a) the loners unwilling to join the public goods game, (b) the cooperators ready to join the group and to contribute their effort, and (c) the defectors who join, but do not contribute. Assuming that groups form randomly, the payoffs for the different strategies $P_{c}, P_{d}$ and $P_{l}$ are then determined by the relative frequencies $x, y$ and $z$ of the three strategies.

\section{The Equations of Motion}

Evolutionary game theory assumes that a strategy's payoff determines the growth rate of its frequency within the population. More precisely, following (Weibull (1995), Schlag (1998) and Hofbauer \& Sigmund (1998), we postulate in our model that players using strategies $i=1, \ldots, n$ occasionally compare their payoff with that of a randomly chosen "model" member of the population, and adopt the strategy of their model with a probability proportional to the difference between the model's payoff and their own, if this is positive (and with probability 0 otherwise). In the continuous time model, the evolution of the frequencies $x_{i}$ of the strategies $i$ is given by

$$
\dot{x}_{i}=\sum_{j} x_{i} x_{j}\left(P_{i}-P_{j}\right)
$$

with $1 \leqslant i, j \leqslant n$, which reduces to the replicator equation

$$
\dot{x}_{i}=x_{i}\left(P_{i}-\bar{P}\right),
$$


where $\bar{P}=\sum x_{j} P_{j}$ is the average payoff in the population.

To be precise, we consider a very large, wellmixed population with three types of players: loners, cooperators and defectors. From time to time, sample groups of $N$ players are randomly chosen and offered to participate in a single public goods game. Note that in large populations, the probability that two players ever encounter again can be neglected. Depending on their type, players either refuse to participate or join the public goods game. In the latter case, they either defect, or cooperate. But, their strategies are specified beforehand, and do not depend on the composition of the sampled group. In particular, we do not consider conditional strategies of the type: cooperate if and only if the group contains more than two cooperators, or the like.

Each player is sampled a number of times, and obtains an average payoff which depends on his strategy as well as the composition of the entire population. This composition changes according to the replicator dynamics [see eqns (3) and (4)]. The intuition behind the dynamics is that occasionally - and independently of the sampling of the public goods teams - a randomly chosen player $A$ compares his or her payoff with that of another player $B$ (also randomly chosen, within the entire population and not limited to the "public goods" groups to which $A$ belonged), and adopts the strategy of $B$, if it yields a higher payoff, with a probability proportional to the difference in their payoffs. Opportunities for updating, i.e. changing the strategy, are supposed to occur much less frequently than opportunities to play in a public goods group. We emphasize that there are other reasonable game dynamics, but for the replicator equation we can produce a full analysis.

For simplicity and without loss of generality, we set the cost $c$ of cooperation equal to 1 . The payoff for loners is then given by the constant

$$
P_{l}=\sigma
$$

In order to compute the payoff values for cooperators and defectors, we first derive the probability that $S$ of the $N$ sampled individuals are actually willing to join the public goods game. In the case $S=1$ (no co-player shows up) we assume that the player has no other option than to play as a loner, and obtains payoff $\sigma$. This happens with probability $z^{N-1}$. For a given player willing to join the public goods game, the probability of finding, among the $N-1$ other players in the sample, $S-1$ co-players joining the group $(S>1)$, is

$$
\left(\begin{array}{c}
N-1 \\
S-1
\end{array}\right)(1-z)^{S-1} z^{N-S} .
$$

The probability that $m$ of these players are cooperators, and $S-1-m$ defectors, is

$$
\left(\frac{x}{x+y}\right)^{m}\left(\frac{y}{x+y}\right)^{S-1-m}\left(\begin{array}{c}
S-1 \\
m
\end{array}\right) .
$$

In that case, the payoff for defectors is $\mathrm{rm} / S$. Hence the expected payoff for a defector in a group of $S$ players $(S=2, \ldots, N)$ is

$$
\begin{aligned}
& \frac{r}{S} \sum_{m=0}^{S-1} m\left(\frac{x}{x+y}\right)^{m}\left(\frac{y}{x+y}\right)^{S-1-m}\left(\begin{array}{c}
S-1 \\
m
\end{array}\right) \\
& =\frac{r}{S}(S-1) \frac{x}{x+y} .
\end{aligned}
$$

Thus,

$$
\begin{aligned}
P_{d}=\sigma z^{N-1}+r \frac{x}{1-z} \sum_{S=1}^{N}\left(\begin{array}{c}
N-1 \\
S-1
\end{array}\right) & (1-z)^{S-1} z^{N-S}\left(1-\frac{1}{S}\right) \\
= & \sigma z^{N-1}+r \frac{x}{1-z} \\
& {\left[1-\sum_{S=1}^{N}\left(\begin{array}{c}
N-1 \\
S-1
\end{array}\right)(1-z)^{S-1} z^{N-S} \frac{1}{S}\right] }
\end{aligned}
$$

and using $\left(\begin{array}{c}N-1 \\ S-1\end{array}\right)=\left(\begin{array}{c}N \\ S\end{array}\right) S / N$ leads to

$$
P_{d}=\sigma z^{N-1}+r \frac{x}{1-z}\left(1-\frac{1-z^{N}}{N(1-z)}\right)
$$


In a group with $S-1$ co-players playing the public goods game, switching from cooperation to defection yields $1-r / S$. Hence,

$P_{d}-P_{c}=\sum_{S=2}^{N}\left(1-\frac{r}{S}\right)\left(\begin{array}{c}N-1 \\ S-1\end{array}\right)(1-z)^{S-1} z^{N-S}$.

Using the same arguments as before, we obtain

$P_{d}-P_{c}=1+(r-1) z^{N-1}-\frac{r}{N} \frac{1-z^{N}}{1-z}=: F(z)$.

The advantage of defectors over cooperators depends only on the fraction of individuals actually willing to play, i.e. on the fraction of loners $z$. At the same time, it is independent of the loner's payoff $\sigma$.

The sign of $P_{d}-P_{c}$ in fact determines whether it pays to switch from cooperation to defection or vice versa, $F(z)=0$ being the equilibrium condition. We claim that for $r \leqslant 2, F$ has no root, and for $r>2$ exactly one root $\hat{z}$ in the interval $(0,1)$. In order to show this, we consider the function $G(z)=F(z)(1-z)$ which has the same roots as $F(z)$ in $(0,1)$ and note that (a) $G(0)=$ $1-r / N>0, \quad$ (b) $G(1)=0, \quad$ (c) $\quad G(z) \asymp(2-r)$ $(N-1)(1-z)^{2}$ for $z \rightarrow 1$, such that in a neighborhood of $z=1 G(z)$ is negative for $r>2$, and (d) $G^{\prime \prime}(z)=z^{N-3}(N-1)((N-2)(r-1)-z(N r-$ $N-r)$ ) changes sign at most once in $(0,1)$. Thus, for $r>2$ [which by eqn (1) implies $N>2$ ] there exists a threshold value of the loners frequency $\hat{z}$ above which cooperators fare better than defectors (see Fig. 1).

The average population payoff $\bar{P}$ can now be rewritten using the condition $y=1-x-z$ :

$$
\begin{aligned}
\bar{P} & =x P_{c}+y P_{d}+z P_{l} \\
& =x\left(P_{c}-P_{d}\right)+z\left(\sigma-P_{d}\right)+P_{d} \\
& =-x\left(P_{d}-P_{c}\right)+(1-z)\left(P_{d}-\sigma\right)+\sigma .
\end{aligned}
$$

Substituting eqns (5) and (6) then yields

$$
\bar{P}=\sigma-[(1-z) \sigma-(r-1) x]\left(1-z^{N-1}\right) .
$$

\section{The Dynamics}

Let us now analyse the replicator dynamics. The corners of the simplex $S_{3}=\{(x, y, z)$ :

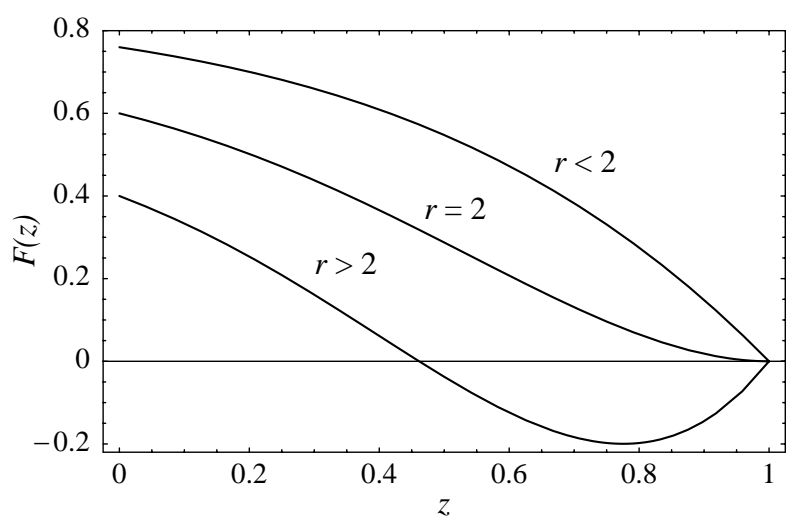

FIG. 1. The difference between the payoff of cooperators $P_{c}$ and defectors $P_{d}$ is a function of the fraction of loners $z: F(z)=P_{d}-P_{c}$. If almost everybody is participating in the public goods game $(z \rightarrow 0)$ then $F(z)>0$ holds and it pays to defect. However, for interest rates $r>2$, if the proportion $z$ of loners increases, it eventually pays to cooperate $(F(z)<0)$ and the social dilemma disappears - at least for a while. $F(z)$ has either no or a unique root in the interval $(0,1)$.

$x, y, z \geqslant 0, x+y+z=1\}$, i.e. the vectors $\mathbf{e}_{i}$ of the standard basis $(i=c, d, l$ in a straightforward notation), are obviously fixed points. There are no other fixed points on the boundary of $S_{3}$. In fact, the edge $\mathbf{e}_{c} \mathbf{e}_{d}$ consists of an orbit leading from $\mathbf{e}_{c}$ (cooperators only) to $\mathbf{e}_{d}$ (defectors only), the edge $\mathbf{e}_{d} \mathbf{e}_{l}$ is an orbit leading to the state consisting of loners only, and the orbit $\mathbf{e}_{l} \mathbf{e}_{c}$ closes this heteroclinic cycle of rock-scissors-paper type on the boundary.

In order to analyse the dynamics in the interior, it is useful to show that the replicator equation, defined on the simplex $S_{3}$, can be rewritten in the form of a Hamiltonian system, and thus admits an invariant of motion. Indeed, defining as a new variable $f=x /(x+y)$, i.e. the fraction of cooperators among the individuals actually participating in the public goods game, we obtain

$$
\dot{f}=\frac{y \dot{x}-x \dot{y}}{(x+y)^{2}}=\frac{x y}{(x+y)^{2}}\left(P_{c}-P_{d}\right) .
$$

This, as well as substituting eqn (7) into the replicator equation $\dot{z}=z(\sigma-\bar{P})$, yields

$$
\dot{f}=-f(1-f) F(z),
$$

$$
\dot{z}=[\sigma-f(r-1)] z(1-z)\left(1-z^{N-1}\right)
$$


with $(f, z)$ on the unit square $(0,1)^{2}$. Dividing the right-hand side by the function $f(1-f) z$ $(1-z)\left(1-z^{N-1}\right)$, which is positive on the unit square, corresponds to a change in velocity which does not affect the orbits. This yields

$$
\begin{gathered}
\dot{f}=\frac{-F(z)}{z(1-z)\left(1-z^{N-1}\right)}=:-g(z), \\
\dot{z}=\frac{\sigma-f(r-1)}{f(1-f)}=: l(f) .
\end{gathered}
$$

Introducing $H:=G+L$, where $G(z)$ and $L(f)$ are primitives of $g(z)$ and $l(f)$ :

$$
G(z)=\left(1-\frac{r}{N}\right) \log z+\left(\frac{r}{2}-1\right) \log (1-z)+R(z),
$$

$$
L(f)=\sigma \log f+(r-1-\sigma) \log (1-f)
$$

with $R(z)$ bounded on $[0,1]$, we obtain the Hamiltonian system

$$
\begin{gathered}
\dot{f}=-\frac{\partial H}{\partial z}, \\
\dot{z}=\frac{\partial H}{\partial f} .
\end{gathered}
$$

The actual dynamics of the system depends on whether the condition $P_{d}=P_{c}$ can be satisfied in the interior $S_{3}$, and hence on the interest rate $r$. For $r \leqslant 2$ there are no fixed points except the corners and all trajectories in int $S_{3}$ are homoclinic orbits of $\mathbf{e}_{l}$. Thus, the system will display intermittently brief bursts of cooperation, but always ends up with no one willing to participate in the public goods game, as shown in Fig. 2.

For $r>2$, eqn (2) implies that there exists a unique fixed point $\mathbf{Q}=(\hat{x}, \hat{y}, \hat{z})$ in int $S_{3}$ such that $F(\hat{z})=0$ and:

$$
\hat{x}=\frac{\sigma}{r-1}(1-\hat{z})
$$

as well as

$$
\hat{y}=\left(1-\frac{\sigma}{r-1}\right)(1-\hat{z})
$$

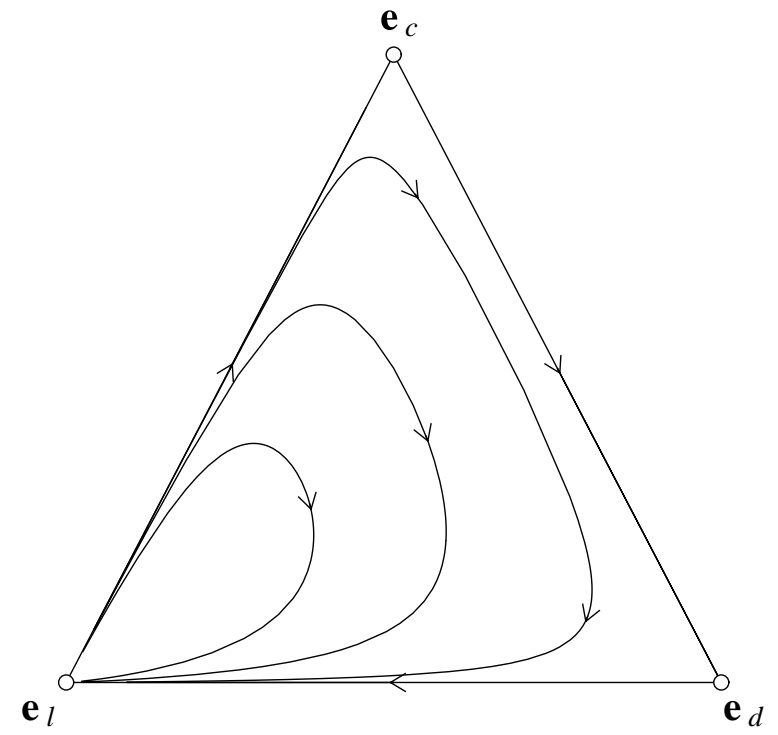

FIG. 2. The three corners $\mathbf{e}_{c}, \mathbf{e}_{d}, \mathbf{e}_{l}$ of $S_{3}$ are saddle points (but $\mathbf{e}_{l}$ is not hyperbolic) and the boundary bd $S_{3}$ represents a rock-scissors-paper type heteroclinic cycle. For small interest rates, $r<2$, no fixed point exists in int $S_{3}$ and all orbits converge to $\mathbf{e}_{l}$. But $\mathbf{e}_{l}$ is not Lyapunov stable. Parameters: $N=5, r=1.8, \sigma=0.5$.

which follows from $P_{d}=P_{l}$. Due to the fact that the system is conservative, and the Hamiltonian $H$ attains a strict (global) maximum at $(\sigma /$ $(r-1), \hat{z})$, the interior equilibrium $\mathbf{Q}$ is a center, i.e. it is neutrally stable and surrounded by closed orbits (see Fig 3). Actually, all interior orbits are closed: eqn (10) shows that $G(z) \rightarrow$ $-\infty$ for $z \rightarrow 0,1$ if $2<r<N$, and eqn (11) implies that $L(f) \rightarrow-\infty$ as $f \rightarrow 0,1$ if $\sigma<r-1$. Therefore, $H \rightarrow-\infty$ uniformly near the boundary of $[0,1]^{2}$ and hence all level sets of $H$ are closed curves. In particular, no interior orbit converges to the non-hyperbolic equilibrium $\mathbf{e}_{l}$.

Variations of the three parameters $N, r, \sigma$ allow to position $\mathbf{Q}$ anywhere in the interior of the simplex (see Fig. 4). Note that in general all three parameters must be adjusted to place $\mathbf{Q}$ in a particular location. According to eqns (12) and (13), the fixed point $\mathbf{Q}$ lies on the line

$$
x=\frac{\sigma}{r-1-\sigma} y
$$

independent of the group size $N$. For increasing $N, \mathbf{Q}$ moves towards the corner $\mathbf{e}_{l}$ and in the 


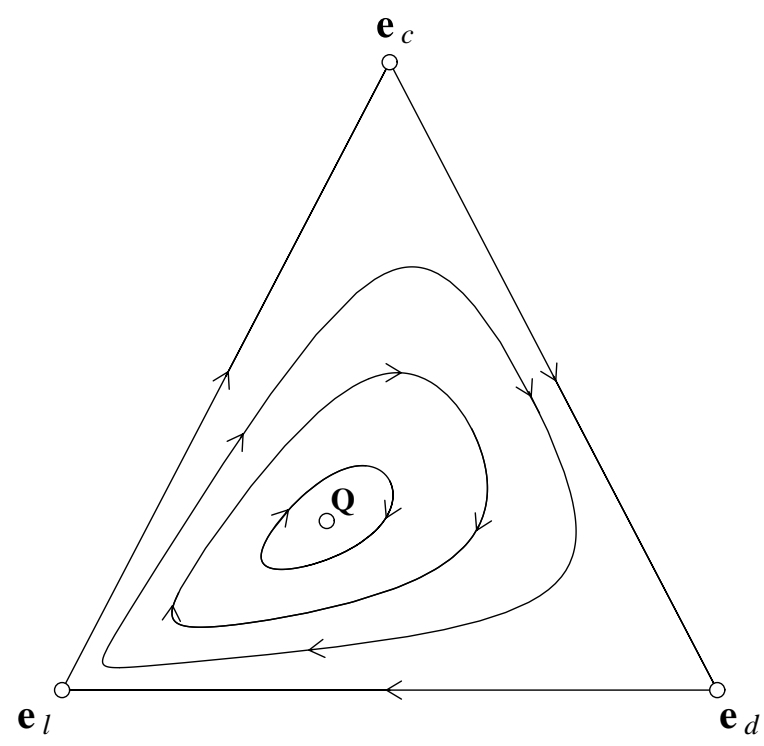

FIG. 3. For $r>2$, the three corners $\mathbf{e}_{c}, \mathbf{e}_{d}, \mathbf{e}_{l}$ are again saddle points and bd $S_{3}$ represents a heteroclinic cycle. In int $S_{3}$ a single fixed point $\mathbf{Q}$ appears. It is a center surrounded by closed orbits (see text). Parameters: $N=5, r=3, \sigma=1$.

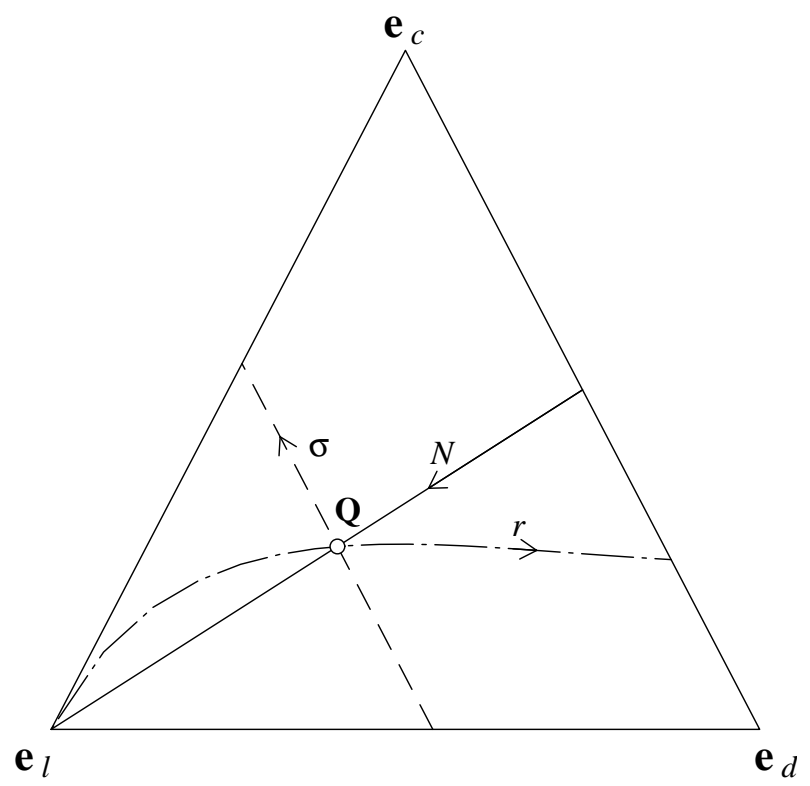

FIG. 4. The position of the center $\mathbf{Q}$ in $S_{3}$ depends on the values of the parameters $N, r$ and $\sigma$. The intersection of the three lines corresponds to $N=5, r=3, \sigma=1$. Each line indicates the displacement of the center when varying a single parameter. Increasing the number of potential participants $N$ shifts the center along the solid line in the direction indicated by the arrow, i.e. towards the corner $\mathbf{e}_{l}$. Similarly, increasing $\sigma$ shifts the center upwards on the dashed line $z=\hat{z}$ and increasing $r$ moves the center to the right, along the dash-dotted line. For $r \rightarrow 2$, the center approaches the corner $\mathbf{e}_{l}$.

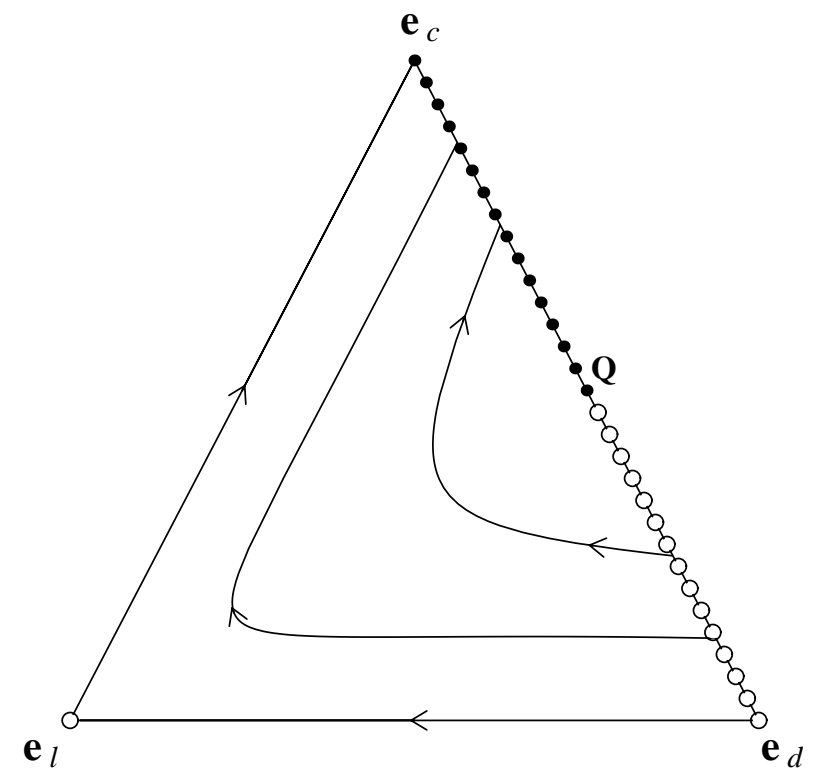

FIG. 5. In the limiting case $r=N$, the edge $\mathbf{e}_{c} \mathbf{e}_{d}$ is a line of fixed points, stable on $\mathbf{e}_{c} \mathbf{Q}$ (closed circles) and unstable on $\mathbf{Q} \mathbf{e}_{d}$ (open circles). Random drift and occasional appearances of the missing loner strategy will eventually drive the system close to the corner $\mathbf{e}_{c}$ with almost everybody cooperating. Parameters: $N=3, r=3, \sigma=1$.

limit $N \rightarrow \infty$ homoclinic orbits issuing from and leading to $\mathbf{e}_{l}$ are obtained.

For the limiting cases $r=N, \sigma=r-1$ and $\sigma=0, \mathbf{Q}$ approaches the edges $\mathbf{e}_{c} \mathbf{e}_{d}, \mathbf{e}_{l} \mathbf{e}_{c}$ or $\mathbf{e}_{l} \mathbf{e}_{d}$, respectively. In particular, for $r=N$, cooperation becomes stable in the sense that, while the state can fluctuate along the edge $z=0$ by random drift, any small fluctuation introducing the missing loners will be offset in such a way that the loners vanish again and the number of cooperators is larger than previously (see Fig. 5).

Although the time averages of the state variables over an orbit of period $T$, defined as $\bar{v}=(1 / T) \int_{0}^{T} v \mathrm{~d} t$, depend on the initial conditions, the following relations hold for every orbit. First, the average fraction of cooperators among playing individuals corresponds to its value at the equilibrium point $\mathbf{Q}$ :

$$
\frac{\bar{x}}{\bar{x}+\bar{y}}=\frac{\sigma}{r-1} .
$$

This means that the time average lies on the solid line in Fig. 4 which connects $\mathbf{Q}$ and $\mathbf{e}_{l}$. Second, the average of the fraction of cooperators among 
participants in public goods games $\bar{f}$ corresponds to the fraction of the averages:

$$
\bar{f}=\frac{\sigma}{r-1} .
$$

Surprisingly, perhaps, increasing $r$ always favors defection, i.e. it decreases the fraction $f$ of cooperators among those actually engaging in the public goods game.

According to numerical calculations, the time average lies on the line segment $\mathbf{Q e}_{l}$ and converges to $\mathbf{e}_{l}$ as the closed orbit approaches the boundary of $S_{3}$. We can offer only a heuristic explanation of this observation: The closer the periodic orbit is to the boundary the more time it will spend near the degenerate equilibrium $\mathbf{e}_{l}$ (both eigenvalues zero) where motion is much slower than close to the hyperbolic equilibria $\mathbf{e}_{c}$ and $\mathbf{e}_{d}$.

Let us show how eqn (15) is deduced by integrating eqn (9). Remembering that, by definition, $x=f(1-z)$, and dividing both sides of eqn (9) by $z\left(1-z^{N-1}\right)$, we get

$$
\begin{aligned}
& \int_{0}^{T}[\sigma(1-z)-(r-1) x] \mathrm{d} t \\
& =\int_{0}^{T} \frac{\dot{z} \mathrm{~d} t}{z\left(1-z^{N-1}\right)}=p(z) \mid \begin{array}{l}
z(T) \\
z(0)
\end{array}
\end{aligned}
$$

$p(z)$ being a primitive of $\left[z\left(1-z^{N-1}\right)\right]^{-1}$. Since the orbits are closed, the last term vanishes and the proportionality between $\bar{x}$ and $1-\bar{z}$, i.e. $\bar{x}+$ $\bar{y}$ follows. The time average (16) follows in the same way after dividing eqn (9) by $z(1-z)\left(1-z^{N-1}\right)$.

Due to the properties of the replicator equation, the time averages of the payoffs for the three different strategies are equal and reduce to the payoff of loners $\sigma$ :

$$
\bar{P}_{c}=\bar{P}_{d}=\bar{P}_{l}=\sigma .
$$

Thus, in the long run, no one does better or worse than the loners.

\section{Discussion}

The oscillations, and thus the recurrent increase in cooperation, are due to the fact that a public goods game needs not always be a social dilemma. In a public goods game, those players who are defecting are always better off than those players who are cooperating. Nevertheless, if the group size $S$ of participating players is less than the interest rate $r$, it pays the individual player to switch from defection to cooperation. If players have the option of an asocial "fallback solution", they can refuse to join the public goods game. If enough players refuse to join, the group becomes so small that the game is no longer a social dilemma. But then, the higher payoff obtained by the cooperators in the public goods game causes more players to join, and larger groups of public goods players create the temptation to defect, i.e. the social dilemma. This requires $r>2$, a condition which is similar to the condition that in the Prisoner's dilemma game, the benefit exceeds twice the cost: this condition is essential for the stability of the Pavlov strategy (Nowak \& Sigmund, 1995). It may be argued that this condition can also be found in Hamilton's rule for kinship selection. Here, the cost-to-benefit ratio should exceed the degree of relatedness between donor and recipient, but under "normal" conditions (no inbreeding, etc.) this relatedness is at most $1 / 2$.

The proposed model for an optional public goods game represents one of the rare cases where a highly nonlinear system of replicator equations can be fully analysed by purely analytical means. For small interest rates, $r \leqslant 2$, homoclinic orbits are observed starting in and returning to $\mathbf{e}_{l}$, i.e. the state where no one participates in the public goods game. For $r>2$ a fixed point occurs in the interior of the simplex $S_{3}$. By reducing the replicator equations to a Hamiltonian system, we see that $\mathbf{Q}$ is actually a center and that in int $S_{3}$ only closed orbits appear. From this follow various conditions on the time averages of the frequencies and payoffs of the three strategies. For example, the average ratio of cooperators and defectors corresponds to the ratio of the averages and is independent of the initial configuration and the group size $N$. It turns out to be impossible to increase cooperation by increasing the interest rate $r$-on the contrary, it favors defection and lowers $\bar{x} / \bar{y}$. In order to promote cooperation, one should rather increase the loner's payoff $\sigma$ or reduce the group size $N$. Note that in the latter case $\bar{x} / \bar{y}$ still 
increases even when keeping the profits for each invested dollar constant $(r / N=$ const $)$. The fact that cooperation is favored in smaller groups agrees with other theoretical as well as experimental results (Bonacich et al., 1976; Boyd \& Richerson, 1988; Milinski et al., 1990; Hauert \& Schuster, 1998).

We stress that the dynamics obtained in this simple and, we believe, natural model is highly degenerate: it has a center, an invariant of motion, a heteroclinic cycle, a non-hyperbolic fixed point, and an even number of Nash equilibria. All these properties are non-generic under the usual assumptions. But as we show in Hauert et al. (2002), a wide variety of adaptive mechanisms, corresponding to many different types of evolutionary game dynamics, lead to persistent oscillations in the frequencies of the three strategies.

The option to drop out from a public goods game, i.e. a social and economic enterprise, avoids deadlocks in states of mutual defection and economic stalemate. As a prerequisite, the possible gain-i.e. the "interest" $r$ - has to be quite large. The enterprise must offer a considerable advantage. In simple societies, such situations may occur in big game hunting or in war. Small groups of volunteers are known to be efficient for difficult tasks. This must have been known to military people for ages. We only quote Marbot, an officer of Napoleon: "To face immense perils, volunteers are infinitely preferable to bodies of men under orders." Success attracts larger groups of participants, but growth may inherently spell decline. This mechanism leads to oscillations in the composition of the population. However, the average effect on the individual's payoff is just the same as if this possibility did not exist and all members of the population were loners.

Ch.H. acknowledges support of the Swiss National Science Foundation; S.D. acknowledges support of the Department of Mathematics, Universitá Statale di Milano; K.S. acknowledges support of the Wissenschaftskolleg WK W008 Differential Equation Models in Science and Engineering.

\section{REFERENCES}

Batali, J. \& Kitcher, P. (1995). Evolution of altruism in optional and compulsory games. J. theor. Biol. 175, $161-171$.

Binmore, K. G. (1994). Playing Fair: Game Theory and the Social Contract. Cambridge, MA: MIT Press.

Bonacich, P., Sure, G. H., Kahan, J. P. \& Meeker, R. J. (1976). Cooperation and group size in the $n$-person prisoner's dilemma. J. Conflict Resolut. 20, 687-705.

BOyd, R. \& Richerson, P. J. (1988). The evolution of reciprocity in sizeable groups. J. theor. Biol. 132, 337-356.

Dawes, R. M. (1980). Social dilemmas. Ann. Rev. Psychol. 31, 169-193.

Dugatkin, L. A. (1997). Cooperation Among Animals: an Evolutionary Perspective. Oxford: Oxford University Press.

Fehr, E. \& GÄChter, S. (2002). Altruistic punishment in humans. Nature 415, 137-140.

Hamilton, W. D. (1963). The evolution of altruistic behaviour. Am. Nat. 97, 354-356.

Hauert, C. \& Schuster, H. G. (1997). Effects of increasing the number of players and memory size in the iterated prisoner's dilemma: a numerical approach. Proc. R. Soc. Lond. B 264, 513-519.

Hauert, C. \& Schuster, H. G. (1998). Extending the iterated prisoner's dilemma without synchrony. $J$. theor. Biol. 192, 155-166.

Hauert, C., De Monte, S., Hofbauer, J. \& Sigmund, K. (2002). Volunteering as Red Queen mechanism for co-operation in public goods games. Science, 296, 1129-1132.

Hofbauer, J. \& Sigmund, K. (1998). Evolutionary Games and Population Dynamics. Cambridge: Cambridge University Press.

Kagel, J. H. \& Roth, A. E., eds (1995). The Handbook of Experimental Economics. Princeton, NJ: Princeton University Press.

Milinski, M., Pfluger, D., Külling, D. \& Kettler, R. (1990). Do sticklebacks cooperate repeatedly in reciprocal pairs? Behav. Ecol. Sociobiol. 27, 17-21.

NowaK, M. A. \& Sigmund, K. (1995). Invasion dynamics of the finitely repeated prisoner's dilemma. Games Econ. Behav. 11, 364-390.

Orbell, J. H. \& Dawes, R. M. (1993). Social welfare, cooperators' advantage, and the option of not playing the game. Am. Soc. Rev. 58, 787-800.

ScHlaG, K. (1998). Why imitate, and if so, how? A bounded rational approach to multi-armed bandits. J. Econ. Theory 78, 130-156.

Trivers, R. L. (1971). The evolution of reciprocal altruism. Q. Rev. Biol. 46, 35-57.

Weibull, J. W. (1995). Evolutionary Game Theory. Cambridge, MA: MIT Press.

Wilson, D. S. \& Sober, E. (1994). Reintroducing group selection to the human behavioral sciences. Behav. Brain Sci. 17, 585-654. 